\title{
Pretransplant malnutrition, inflammation, and atherosclerosis affect cardiovascular outcomes after kidney transplantation
}

\author{
Jin Ho Hwang ${ }^{1}$, Jiwon Ryu ${ }^{2}$, Jung Nam An ${ }^{3}$, Clara Tammy Kim ${ }^{4}$, Hyosang Kim ${ }^{5}$, Jaeseok Yang ${ }^{6}$, Jongwon Ha ${ }^{6}$, \\ Dong Wan Chae ${ }^{7}$, Curie Ahn ${ }^{6,8}$, In Mok Jung ${ }^{9}$, Yun Kyu Oh ${ }^{3}$, Chun Soo Lim³ ${ }^{3}$ Duck-Jong Han ${ }^{10}$, Su-Kil Park ${ }^{5}$, \\ Yon Su Kim ${ }^{8}$, Young Hoon Kim ${ }^{10^{*} \dagger}$ and Jung Pyo Lee ${ }^{3^{*} \dagger}$
}

\begin{abstract}
Background: Malnutrition, inflammation, and atherosclerosis (MIA) syndrome is associated with a high mortality rate in patients with end-stage renal disease. However, the clinical relevance of MIA syndrome in kidney transplantation (KT) recipients remains unknown.

Methods: We enrolled 1348 adult KT recipients. Recipients were assessed based on serum albumin, cholesterol, or body mass index for the malnutrition factor and C-reactive protein level for the inflammation factor. Any history of cardiovascular (CV), cerebrovascular, or peripheral vascular disease satisfied the atherosclerosis factor. Each MIA factors were assessed by univariate analysis and we calculated an overall risk score by summing up scores for each independent variable. The enrolled patients were divided into 4 groups depending on the MIA score $(0,2-4,6,8-10)$.

Results: The patients with higher MIA score showed worse outcome of fatal/non-fatal acute coronary syndrome (ACS) $(p<0.001)$ and composite outcomes of ACS and all-cause mortality $(p<0.001)$ than with the lower MIA score. In multivariate analysis, ACS showed significantly higher incidence in the MIA score 8-10 group than in the MIA score 0 group (Hazard ratio $6.1295 \%$ Confidence interval 1.84-20.32 $p=0.003$ ).
\end{abstract}

Conclusions: The presence of MIA factors before $K T$ is an independent predictor of post-transplant CV outcomes.

Keywords: Acute Coronary Syndrome, Atherosclerosis, Cardiovascular Outcome, Inflammation, Kidney

Transplantation, Malnutrition

\section{Background}

Cardiovascular disease (CVD) is the main cause of morbidity and mortality in patients with end-stage renal disease (ESRD) despite major research efforts and improvements in dialysis technology [1]. There have been many discussions about traditional risk factors that may not sufficiently predict CVD occurrence in patients with ESRD [2-5].

Malnutrition, inflammation, and atherosclerosis (MIA) syndrome is associated with a high mortality rate and

\footnotetext{
* Correspondence: kyh-001@hanmail.net; nephrolee@gmail.com

${ }^{\dagger}$ Equal contributors

${ }^{10}$ Department of Surgery, Asan Medical Center and University of Ulsan College of Medicine, Seoul, South Korea

${ }^{3}$ Department of Internal Medicine, Seoul National University Boramae Medical Center, Seoul, South Korea

Full list of author information is available at the end of the article
}

increased cardiovascular event rate in patients with ESRD [6]. The 3 factors of MIA syndrome interact with each other and create a vicious cycle [7]. Malnutrition or protein-energy wasting may aggravate existing inflammation, accelerating atherosclerosis and increasing susceptibility to infection $[8,9]$. Chronic inflammation is common in patients with chronic kidney disease, in part because of the decreased glomerular filtration rate (GFR), and also because of the dialysis procedure [10, 11]. Inflammation plays a key role in atherosclerosis and may contribute to an increased cardiovascular mortality associated with endothelial dysfunction and increased oxidative stress $[9,12]$. With these observations, the term malnutrition inflammation complex syndrome (MICS) was coined. 
Kidney transplantation provides a better quality of life for patients with ESRD. With the development and advancement of immunosuppressive agents, renal allograft survival rates have improved over the years. Even after the transplantation, CVD is an important cause of death [13]. Moreover, the immunosuppressive agents increase atherosclerotic risk by elevating blood pressure and by aggravating several metabolic profiles such as dyslipidemia and new onset diabetes after transplantation (NODAT) [13, 14].

While several studies about MICS or MIA syndrome in patients with ESRD have been reported [6, 7, 9, 15], only a few reports have evaluated transplant wait-listed patients with ESRD and the association between pretransplant parameters (e.g., C-reactive protein (CRP), albumin) and post-transplant outcomes [16-18]. Thus, the clinical relevance of MIA syndrome in kidney transplant recipients remains unclear. We hypothesized that MIA syndrome is associated with poorer post-transplant outcome.

\section{Methods}

\section{Ethics statement}

This study was approved by the institutional review board at Seoul National University Hospital (H-1302018-462), and the need for informed consent from the patients was waived because of the retrospective study design. All clinical investigations were conducted in accordance with the guidelines of the 2008 Declaration of Helsinki.

\section{Study design and patients}

This study was performed as a retrospective, multicenter study. Among the patients in whom kidney transplantation had been performed at Seoul National University Hospital, Seoul National University Boramae Medical Center, Seoul National University Bundang Hospital, and Asan Medical Center from Jun. 1999 through Dec. 2011, we reviewed the medical records of 2425 individuals and collected data from 1348 patients. All patients were adults (age $\geq 15$ years); had pretransplant CRP, serum albumin, and cholesterol data available; received renal transplants; and were followed for more than one year after transplantation (Fig. 1). Patients with a previous transplantation history and those with unavailable pretransplant laboratory profiles were excluded. Patients with a follow-up duration less than one year were also excluded from the analysis.

Clinical parameters such as age at the time of kidney transplantation, sex, body mass index (BMI), laboratory test results (CRP, serum albumin, cholesterol, intact patathyroid hormone (PTH), and cytomegalovirus (CMV) immunoglobulin G (IgG) status), underlying diseases (hypertension, diabetes mellitus (DM), ischemic heart disease, liver cirrhosis, and tuberculosis), dialysis modality, and duration of dialysis before transplantation were collected. Donor factors such as age at transplantation, sex, donor source, and CMV IgG status were evaluated.

\section{Definitions and grouping}

We evaluated enrolled patients by defining and calculating MIA factors. Between patients with or without acute coronary syndrome (ACS), the univariate Cox regression was performed to identify the influence of variables such as serum albumin, cholesterol, and BMI for malnutrition factor, and CRP for inflammation factor. Researchers considered the atherosclerosis factor to be satisfied if there was any history of $\mathrm{CV}$, cerebrovascular, or peripheral vascular disease. Recipients were assessed according to the quartile value of pretransplant albumin and CRP. We evaluated each independent variable for the assumption of proportional hazards. From the regression model, the $\beta$-coefficient of each variable was assigned score by rounding to the nearest integer [19]. After that, we calculated an overall risk score by summing up the scores for each independent predictor variable for a given patient. Subsequently, the enrolled patients were divided into four groups depending on the MIA score considering score homogeneity $(0,2-4,6,8-10)$.

Researchers have checked cholesterol, albumin, and CRP levels at least two time points within one year before transplantation and used mean value of the each variables. The minimum interval between the two time points was four weeks.

The cardiovascular outcome of fatal/non-fatal ACS was defined as usual ACS, which includes unstable angina/ non-ST elevated myocardial infarction (MI) and ST elevated MI. In this study all the patients with ACS were confirmed by coronary angiography.

\section{Primary and secondary objectives}

The primary objective of the study was to evaluate whether pretransplant MIA factors affect the occurrence of fatal/non-fatal ACS after transplantation. The secondary objective of the study was to evaluate the composite of ACS with all-cause mortality and graft failure. Graft failure was defined as a requirement for maintenance dialysis or re-transplantation.

\section{Statistical analysis}

All analyses and calculations were performed by using the IBM SPSS Statistics V21.0 (IBM Corporation, Armonk, NY, USA). Continuous variables were expressed as the mean \pm standard deviation and as the percentage for categorical variables. Continuous and categorical data were compared by using the Student's $t$-test and the chi-square test, respectively. The ACS-free survival rates and other event-free survival rates were calculated by using the 


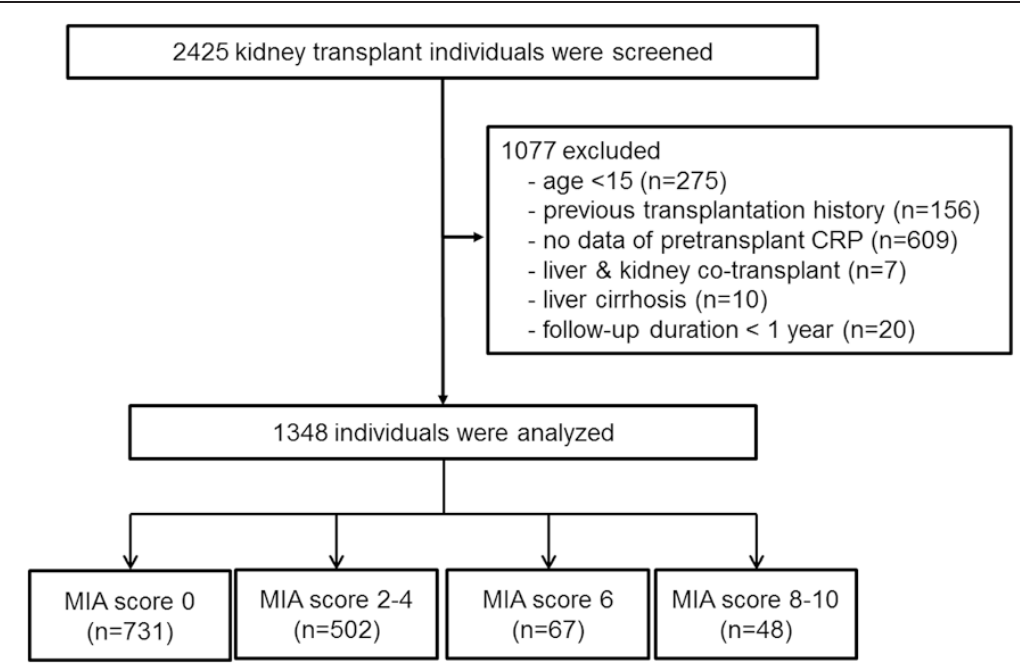

Fig. 1 Defining the study population. We reviewed the medical records of 2425 individuals and collected data from 1348 patients

Kaplan-Meier method, and comparison between groups was performed by using the log-rank test. The Cox regression model was used to identify risk factors and to calculate the hazard ratio (HR) and $95 \%$ confidence interval (CI). The corresponding $\beta$-coefficient for each independent variable from the regression model for the occurrence of ACS was used to calculate MIA score. Differences with $p<0.05$ were considered statistically significant.

\section{Results}

\section{Baseline characteristics of enrolled patients}

The baseline characteristics of the 1348 enrolled patients are described in Table 1. The mean age of patients at transplantation was 43.0 years, and $57.2 \%$ were men $(n=771)$. The mean follow-up duration was $57.0 \pm$ 36.0 months. The pretransplant BMI, albumin, CRP, and cholesterol levels were $22.4 \pm 3.2 \mathrm{~kg} / \mathrm{m}^{2}, 3.7 \pm 0.5 \mathrm{~g} / \mathrm{dL}$, $0.48 \pm 1.17 \mathrm{mg} / \mathrm{dL}$, and $158.8 \pm 40.9 \mathrm{mg} / \mathrm{dL}$, respectively. Hypertension was diagnosed in 1198 patients (88.9\%) and DM was diagnosed in 275 patients (20.4 \%). Onehundred fifteen patients ( $8.5 \%$ ) had experienced at least one of the following vascular diseases: CVD, cerebrovascular disease, or peripheral vascular disease. There were more patients with dyslipidemia in the higher MIA score group (63.8 \% in MIA score 8-10 group, $26.6 \%$ in average). There was no significant difference in total cholesterol level between the patients who took the lipid lowering agent and the patients who did not (156.9 \pm 45.8 vs. $159.4 \pm 38.8, p=0.38$, data not shown). Preemptive kidney transplantation was performed in 217 patients (16.1\%), and hemodialysis was performed frequently before transplantation ( $n=679,60.0 \%$ of dialysis patients). Most enrolled patients received only kidney transplantation $(n=1211,89.8 \%)$, and most grafts were received from living donors $(n=1065,79.0 \%)$. The prescription of immunosuppressive agents after transplant was not different between the groups.

\section{Pretransplant MIA factor profile}

The HR for cardiovascular disease of albumin, CRP, and previous history of vascular disease was 2.040, 1.893, and 6.41 respectively. The corresponding scores for the same MIA factors were 2, 2, and 6 (Table 2). Sevenhundred thirty-one patients (54.2 \%) did not satisfy any of the MIA factors (MIA score 0). Three-hundred ninety-eight patients $(29.5 \%)$ had one MIA factor with score 2 and 67 patients $(5.0 \%)$ had 1 MIA factor with score 6. Nine patients had all of three MIA factors (score 10). Other MIA score groups and each component are shown in Table 3. The distribution of MIA scores of the patients with pre-emptive transplantation was not different with the patients who underwent dialysis before transplantation ( $p=0.451$, data not shown).

\section{Factors related to the occurrence of ACS}

Univariate analysis of the association between ACS and covariates showed a significant relationship with age (HR, 1.08; $95 \% \mathrm{CI}, 1.03-1.12 ; p=0.001$ ); male sex (HR, 3.54; $95 \% \mathrm{CI}, 1.45-8.60 ; p=0.005)$; BMI (HR, 1.11; $95 \% \mathrm{CI}, 1.00-1.23 ; p=0.049)$; DM (HR, 3.55; $95 \% \mathrm{CI}$, $1.78-7.05 ; p<0.001)$; deceased donor (HR, 2.72; $95 \%$ CI, $1.12-6.61 ; p=0.027)$; NODAT $(\mathrm{HR}, 2.80 ; 95 \% \mathrm{CI}$, $1.24-6.32 ; p=0.013)$; previous history of vascular disease (HR, 6.41; $95 \% \mathrm{CI}, 3.09-13.33 ; p<0.001$ ); and the MIA score group (10 vs. 0; HR, 32.96; $95 \%$ CI, 7.30-148.94; $p<0.001$ and $8-10$ vs. 0 ; HR, 11.18 ; $95 \% \mathrm{CI}, 4.12-$ 30.33; $p<0.001$ ). Of these, MIA score $8-10$ (HR, 6.12; $95 \%$ CI, 1.84-20.32; $p=0.003)$; age (HR, 1.05; $95 \% \mathrm{CI}$, $1.01-1.10 ; p=0.008)$; NODAT (HR, 2.88 ; $95 \%$ CI, $1.03-$ 8.10; $p=0.045$ ); albumin (HR, 2.33; $95 \% \mathrm{CI}, 1.11-4.88$; 
Table 1 Baseline characteristics of the study subjects

\begin{tabular}{|c|c|c|c|c|c|c|}
\hline & $\begin{array}{l}\text { MIA score } 0 \\
(n=731)\end{array}$ & $\begin{array}{l}\text { MIA score } 2-4 \\
(n=502)\end{array}$ & $\begin{array}{l}\text { MIA score } 6 \\
(n=67)\end{array}$ & $\begin{array}{l}\text { MIA score } 8-10 \\
(n=48)\end{array}$ & $\begin{array}{l}\text { Total } \\
(N=1348)\end{array}$ & p-value \\
\hline Age at the transplantation ${ }^{a}$ & $41.1 \pm 11.9$ & $42.8 \pm 12.0$ & $52.5 \pm 10.4$ & $57.7 \pm 8.0$ & $43.0 \pm 12.3$ & $<0.001^{\ddagger}$ \\
\hline Recipient's sex (male, \%) & 54.7 & 57.4 & 67.2 & 79.2 & 57.2 & 0.003 \\
\hline Body weight $(\mathrm{kg})^{\mathrm{a}}$ & $59.9 \pm 10.7$ & $61.4 \pm 12.0$ & $62.4 \pm 11.0$ & $64.8 \pm 9.0$ & $60.8 \pm 11.2$ & $0.003^{\ddagger}$ \\
\hline Height $(\mathrm{cm})^{a}$ & $164.2 \pm 8.6$ & $164.4 \pm 8.8$ & $164.4 \pm 7.8$ & $166.4 \pm 7.4$ & $164.4 \pm 8.6$ & 0.374 \\
\hline $\operatorname{BMI}\left(\mathrm{kg} / \mathrm{m}^{2}\right)^{\mathrm{a}}$ & $22.1 \pm 2.9$ & $22.6 \pm 3.5$ & $23.0 \pm 3.3$ & $23.3 \pm 2.6$ & $22.4 \pm 3.2$ & $0.001^{\S}$ \\
\hline Current smoker (\%) & 10.6 & 9.1 & 23.3 & 6.4 & 9.8 & $<0.001$ \\
\hline \multicolumn{7}{|l|}{ Past medical history } \\
\hline Hypertension (\%) & 88.5 & 87.3 & 100.0 & 95.8 & 88.9 & 0.007 \\
\hline DM (\%) & 14.1 & 21.5 & 49.3 & 64.6 & 20.4 & $<0.001$ \\
\hline Vascular diseases $(\%)^{\mathrm{b}}$ & 0.0 & 0.0 & 100.0 & 100.0 & 8.5 & $<0.001$ \\
\hline Dyslipidemia (\%) & 21.4 & 29.1 & 35.0 & 63.8 & 26.6 & $<0.001$ \\
\hline Renal disease causing ESRD & & & & & & 0.033 \\
\hline DM (\%) & 17.4 & 19.4 & 14.3 & 12.2 & 17.8 & \\
\hline Hypertension (\%) & 10.0 & 9.7 & 17.9 & 12.2 & 10.4 & \\
\hline Glomerulonephritis (\%) & 30.5 & 28.4 & 21.4 & 22.0 & 29.0 & \\
\hline PKD (\%) & 5.3 & 3.9 & 14.3 & 4.9 & 5.2 & \\
\hline Others (\%) & 36.8 & 38.6 & 32.1 & 48.7 & 37.6 & \\
\hline \multicolumn{7}{|l|}{ Laboratory Findings } \\
\hline Serum albumin $(g / d l)^{a}$ & $3.9 \pm 0.4$ & $3.4 \pm 0.5$ & $3.9 \pm 0.3$ & $3.3 \pm 0.6$ & $3.7 \pm 0.5$ & $<0.001^{\ddagger \S}$ \\
\hline $\operatorname{CRP}(\mathrm{mg} / \mathrm{dl})^{\mathrm{a}}$ & $0.11 \pm 0.11$ & $0.97 \pm 1.62$ & $0.15 \pm 0.13$ & $1.25 \pm 2.46$ & $0.48 \pm 1.17$ & $<0.001^{\neq \S}$ \\
\hline Total cholesterol $(\mathrm{mg} / \mathrm{dl})^{\mathrm{a}}$ & $161.6 \pm 37.5$ & $155.7 \pm 44.9$ & $156.5 \pm 38.4$ & $151.6 \pm 48.5$ & $158.8 \pm 40.9$ & 0.044 \\
\hline Triglyceride $(\mathrm{mg} / \mathrm{dl})^{\mathrm{a}}$ & $120.8 \pm 67.2$ & $125.9 \pm 99.2$ & $135.6 \pm 89.5$ & $133.6 \pm 72.9$ & $123.9 \pm 81.1$ & 0.419 \\
\hline LDL cholesterol $(\mathrm{mg} / \mathrm{dl})^{\mathrm{a}}$ & $91.5 \pm 32.1$ & $91.3 \pm 31.8$ & $87.2 \pm 31.6$ & $93.6 \pm 43.2$ & $91.3 \pm 32.4$ & 0.772 \\
\hline Intact PTH (pg/ml) & $262.2 \pm 312.8$ & $238.7 \pm 242.7$ & $181.8 \pm 183.8$ & $249.7 \pm 273.3$ & $249.6 \pm 279.4$ & 0.29 \\
\hline CMV IgG positive (\%) & 93.1 & 96.0 & 92.9 & 96.3 & 94.8 & 0.475 \\
\hline Dialysis before TPL (\%) & 82.2 & 85.7 & 88.1 & 85.4 & 83.9 & 0.491 \\
\hline Preemptive TPL (\%) & 17.8 & 14.3 & 11.9 & 14.6 & 16.1 & 0.491 \\
\hline HD:PD:both or switch (\%) & 60.7:18.4:3.1 & 57.8:24.0:3.9 & 63.3:18.3:6.5 & 68.8:14.6:2.0 & $60.0: 20.4: 3.5$ & 0.308 \\
\hline Dialysis duration (months) & $26.7 \pm 38.8$ & $27.7 \pm 38.0$ & $38.4 \pm 50.8$ & $21.2 \pm 27.7$ & $27.4 \pm 38.8$ & 0.098 \\
\hline \multicolumn{7}{|l|}{ Donor information } \\
\hline Deceased donor (\%) & 20.1 & 21.3 & 29.9 & 17.0 & 21.0 & 0.003 \\
\hline Donor's age (year) ${ }^{a}$ & $40.2 \pm 11.9$ & $40.0 \pm 12.4$ & $41.7 \pm 13.3$ & $43.0 \pm 12.8$ & $40.3 \pm 12.2$ & 0.303 \\
\hline
\end{tabular}

$$
\text { DM (\%) }
$$

$$
\text { PKD (\%) }
$$

aboratory Findings

Total cholesterol $(\mathrm{mg} / \mathrm{dl})^{\mathrm{a}}$

Intact PTH (pg/ml)

HD:PD:both or switch (\%)

onor information

Donor's age (year)
$40.2 \pm 11.9$
$40.0 \pm 12.4$
$41.7 \pm 13.3$

$43.0 \pm 12.8$ 
Table 1 Baseline characteristics of the study subjects (Continued)

\begin{tabular}{|c|c|c|c|c|c|c|}
\hline Donor's sex (male, \%) & 52.5 & 57.7 & 50.0 & 62.5 & 54.6 & 0.178 \\
\hline CMV IgG positive (\%) & 54.9 & 69.9 & 47.8 & 60.4 & 60.3 & $<0.001$ \\
\hline TPL (KT only : SPK : Others, \%) & $89.1: 3.1: 7.8$ & 91.4:4.8:3.8 & $85.1: 4.5: 10.4$ & 89.6:2.1:8.3 & 89.8:3.8:6.4 & 0.053 \\
\hline \multicolumn{7}{|l|}{ Immunosuppressive agents } \\
\hline Steroid maintenance strategy (\%) & 92.6 & 89.5 & 100 & 85.7 & 90.9 & 0.625 \\
\hline CNI (CsA : Tacrolimus, \%) & $34.2: 65.3$ & 35.8:63.6 & 22.8:75.4 & $29.7: 70.3$ & 34.0:65.4 & 0.254 \\
\hline Antimetabolites (Aza : MMF, \%) & $6.5: 88.1$ & 10.2:79.4 & 10.5:80.7 & $8.6: 82.9$ & $8.0: 84.6$ & 0.095 \\
\hline
\end{tabular}

$B M I$ body mass index, DM diabetes mellitus, ESRD end-stage renal disease, $P K D$ polycystic kidney disease, CRP c-reactive protein, $L D L$ low-density lipoprotein, $T P L$ transplantation, $H D$ hemodialysis, $P D$ peritoneal dialysis, $K T$ kidney transplantation, SPK simultaneous pancreas-kidney transplant, CNI calcineurin inhibitor, CsA cyclosporine A, Aza azathioprine, MMF mycophenolate mofetil

a data are expressed as the mean \pm SD

bVascular disease included cardiovascular, cerebrovascular, and peripheral vessel diseases

$\neq_{p}<0.05$ a post hoc andysis betwe MIA score 0 group and MIA score 8-10

$s_{p}<0.05$ at post-hoc ansis beve 
Table 2 Factors associated with the occurrence of ACS

\begin{tabular}{|c|c|c|c|c|c|c|}
\hline & \multicolumn{3}{|c|}{ Univariate } & \multicolumn{3}{|c|}{ Multivariate $^{a}$} \\
\hline & $\mathrm{HR}$ & $95 \% \mathrm{Cl}$ & p-value & $\mathrm{HR}$ & $95 \% \mathrm{Cl}$ & $\mathrm{p}$-value \\
\hline Age & 1.08 & $1.03-1.12$ & 0.001 & 1.05 & $1.01-1.10$ & 0.008 \\
\hline Male & 3.54 & $1.45-8.60$ & 0.005 & 2.26 & $0.89-5.71$ & 0.085 \\
\hline Smoking & 1.92 & $0.77-4.78$ & 0.162 & & & \\
\hline BMI (continuous variable) & 1.11 & $1.00-1.23$ & 0.049 & & & \\
\hline Hypertension & 4.27 & $0.58-31.44$ & 0.154 & - & - & - \\
\hline DM & 3.55 & $1.78-7.05$ & $<0.001$ & 2.58 & $0.99-6.76$ & 0.054 \\
\hline Dialysis before KT (vs. preemptive) & 1.58 & $0.46-5.38$ & 0.469 & - & - & - \\
\hline Dialysis vintage (months) & 1.01 & $0.99-1.013$ & 0.146 & & & \\
\hline Cholesterol (<150 mg/dL) & 1.46 & $0.69-3.07$ & 0.324 & & & \\
\hline Intact PTH & 1.00 & $0.99-1.00$ & 0.384 & & & \\
\hline Recipient CMV IgG (+) & 0.01 & $0.01-20.16$ & 0.998 & & & \\
\hline Donor sex (male) & 0.64 & $0.31-1.29$ & 0.208 & - & - & - \\
\hline Donor age & 1.03 & $0.99-1.06$ & 0.094 & - & - & - \\
\hline Deceased donor (vs. living donor) & 2.72 & $1.12-6.61$ & 0.027 & 2.17 & $0.80-5.91$ & 0.128 \\
\hline Donor CMV IgG (+) & 0.891 & $0.44-1.79$ & 0.745 & & & \\
\hline CsA (vs. tacrolimus) & 1.9 & $0.91-3.95$ & 0.085 & - & - & - \\
\hline NODAT & 2.80 & $1.24-6.32$ & 0.013 & 2.88 & $1.03-8.10$ & 0.045 \\
\hline CMV disease & 0.046 & $0.01-14.33$ & 0.686 & 0.15 & $0.01-41.48$ & 0.712 \\
\hline \multicolumn{7}{|l|}{ MIA factors } \\
\hline Albumin (lowest quartile vs. others) & 2.04 & $0.98-4.24$ & 0.056 & 2.33 & $1.11-4.88$ & 0.026 \\
\hline CRP (highest quartile vs. others) & 1.89 & $0.94-3.82$ & 0.075 & 1.55 & $0.75-3.20$ & 0.237 \\
\hline Previous history of vascular disease ${ }^{a}$ & 6.41 & $3.09-13.33$ & $<0.001$ & 2.61 & $1.08-6.27$ & 0.033 \\
\hline \multicolumn{7}{|l|}{ MIA score ${ }^{a}$} \\
\hline 0 & \multicolumn{3}{|c|}{ Reference } & \multicolumn{3}{|c|}{ Reference } \\
\hline 2 & 1.17 & $0.45-3.05$ & 0.742 & 2.14 & $0.77-5.96$ & 0.147 \\
\hline 4 & 2.51 & $0.79-8.03$ & 0.121 & 1.86 & $0.39-8.73$ & 0.436 \\
\hline 6 & 5.23 & $1.76-15.53$ & 0.003 & 2.93 & $0.90-9.54$ & 0.075 \\
\hline 8 & 7.53 & $2.28-24.85$ & 0.001 & 3.11 & $0.73-13.29$ & 0.125 \\
\hline 10 & 32.96 & $7.30-148.94$ & $<0.001$ & 34.29 & $8.29-141.84$ & $<0.001$ \\
\hline \multicolumn{7}{|l|}{ Group by MIA score ${ }^{a}$} \\
\hline 0 & \multicolumn{3}{|c|}{ Reference } & \multicolumn{3}{|c|}{ Reference } \\
\hline $2-4$ & 1.33 & $0.56-3.16$ & 0.517 & 2.05 & $0.79-5.35$ & 0.142 \\
\hline 6 & 5.28 & $1.78-15.68$ & 0.003 & 3.06 & $0.94-9.99$ & 0.063 \\
\hline $8-10$ & 11.18 & $4.12-30.33$ & $<0.001$ & 6.12 & $1.84-20.32$ & 0.003 \\
\hline
\end{tabular}

Data were analyzed by using the Cox regression, Backward LR method in the multivariate analysis

ACS acute coronary syndrome, DM diabetes mellitus, $K T$ kidney transplantation, NODAT new onset diabetes after transplantation, CsA cyclosporine A, Alb albumin "We performed multivariate analysis about "MIA score", "Previous history of vascular disease", and "Group by MIA score" separately with other variables

$p=0.026)$; and previous history of vascular disease (HR, 2.61; 95 \% CI, 1.08-6.27; $p=0.033$ ) remained significant predictors on multivariate analysis (Table 2). There was no association between ACS and smoking history, hypertension, cholesterol level, intact PTH level, recipient and donor CMV IgG antibody status, donor sex, donor age, dialysis modality before transplantation, immunosuppressive agents, or CMV disease.

\section{ACS based on each MIA factor and impact of ACS on mortality}

There was a significant difference in occurrence of ACS $(p=0.042)$ and composite outcomes $(p=0.016)$ between the lowest quartile and the others when albumin level was analyzed (Fig. 2a, b). Our findings indicate that increased CRP level was not predictive of ACS $(p=0.106)$ or composite outcomes $(p=0.187)$ (Fig. 2c, d). Previous 
Table 3 Crosstable by MIA score group and each component

\begin{tabular}{llllllll}
\hline & $\begin{array}{l}\text { MIA score 0 } \\
(n=731)\end{array}$ & $\begin{array}{l}\text { MIA score 2 } \\
(n=398)\end{array}$ & $\begin{array}{l}\text { MIA score 4 } \\
(n=104)\end{array}$ & $\begin{array}{l}\text { MIA score 6 } \\
(n=67)\end{array}$ & $\begin{array}{l}\text { MIA score 8 } \\
(n=39)\end{array}$ & $\begin{array}{l}\text { MIA score 10 } \\
(n=9)\end{array}$ & $\begin{array}{l}\text { Total } \\
(N=1348)\end{array}$ \\
\hline Albumin lower quartile (\%) $^{a}$ & 0 & $191(48.0)$ & $104(100)$ & 0 & $24(61.5)$ & $9(100)$ & $335(24.3)$ \\
CRP upper quartile (\%) $^{a}$ & 0 & $207(52.0)$ & $104(100)$ & 0 & $15(38.5)$ & $9(100)$ & $340(24.9)$ \\
Atherosclerosis (\%) $^{a}$ & 0 & 0 & 0 & $67(100)$ & $39(100)$ & $9(100)$ & $116(8.5)$ \\
\hline
\end{tabular}

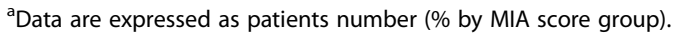

history of vascular disease (any cardiovascular, cerebrovascular, or peripheral vascular disease) was significantly related with ACS $(p<0.001)$ and composite outcomes $(p<0.001)$ (Fig. 2e, F). In 1233 patients without a history of vascular disease, 21 patients experienced ACS after transplantation. However, in 115 patients with a history of vascular disease, 12 patients experienced ACS.

The all-cause mortality was significantly higher in the patients with post-transplant $\mathrm{CV}$ events than in the other patients $(21.2 \%$ vs. $2.5 \%$; $p<0.001)$. Cardiovascular events were the second most common cause of post-transplant mortality in this study population.

\section{MIA score groups and occurrence of ACS}

The patients were divided into 4 groups depending on MIA scores and the ACS, graft outcome, and mortality were evaluated between groups. The ACS occurred in total of 33 patients $(2.4 \%)$. The patients with higher MIA scores showed worse ACS outcomes $(p<0.001$, Fig. 3a) and composite outcomes of ACS and death $(p<0.001$, Fig. 3b) than those in the group with lower MIA scores.

To eliminate the strong effect of vascular disease history, we divided the patients by history of vascular disease and evaluated separately. In patients without previous vascular disease, ACS and composite outcome did not show significant difference according to the quartile value of albumin and CRP (Fig. 4a, c). However, in the patients with a history of vascular disease, ACS $(p=0.002$, Fig. $4 \mathrm{~b})$ and composite outcome $(p=0.011$, Fig. 4d) occurred more in the lowest albumin + highest CRP quartile group than in the other groups.

\section{Other outcomes based on MIA factor}

The overall survival rate was $97.0 \%$, and all-cause mortality rate between the groups did not differ based on MIA score. Infection (23 patients, $56.1 \%$ ) and cardiovascular disease (5 patients, $12.2 \%$ ) were the most common causes of death after transplantation. Eight patients $(19.5 \%)$ died suddenly from unknown cause.

During a mean follow-up duration of $57.0 \pm 36.0$ months, the overall graft survival rate was $94.6 \%$, excluding patients who died with functioning grafts. Graft outcome and all-cause mortality were not different between the groups ( $p=0.973$ and $p=0.183$ ) (Fig. 3c, d). Acute rejection and acute kidney injury related with sepsis was the first and the second most common cause of graft failure ( $23.3 \%$ and $13.7 \%$, respectively). Transplant glomerulopathy $(9.6 \%)$, recurred glomerulonephritis $(6.8 \%)$, noncompliance to immunosuppressive agents (6.8\%), BK virus-induced nephropathy (5.5\%), and renal infarct (4.1\%) were the next common causes. The number of total and graft failure cases in MIA score 6 and 8-10 groups were not enough to perform statistical analysis on the graft failure causes.

The occurrence of CMV disease after KT was not different between the groups, which could be a strong predictor of cardiovascular complications $(7.7 \%, 4.3 \%, 7.1 \%$, and $3.7 \%$ in MIA score $0,2-4,6$, and $8-10 ; p=0.359)$.

\section{Discussion}

In this study of 1348 kidney transplant recipients, we report independent associations of pretransplant MIA score with the occurrence of post-transplant ACS. To date, few reports have examined the association between pretransplant MICS and post-transplant outcomes. Most of these reports focused on different outcomes such as mortality (including cardiovascular mortality and allcause mortality), delayed graft function, chronic allograft nephropathy, or graft outcomes [17, 20-23]. Some were either small in sample size [18] or focused on association only with one MIA factor (albumin or CRP level) [24]. To our knowledge, this is the first multicenter study on the occurrence of ACS in kidney transplant recipients with combined factor for considering pretransplant MIA score.

We defined malnutrition and inflammation arbitrarily in some ways. However, an albumin level $<3.5 \mathrm{mg} / \mathrm{dL}$ was not only a lower quartile value, but also "below normal level". Also a cholesterol level $<150 \mathrm{mg} / \mathrm{dL}$ originated with the malnutrition standards of dialysis patients [25]. In 2003, the American Heart Association and the Centers for Disease Control and Prevention endorsed cutoff points of $<1 \mathrm{mg} / \mathrm{dL}$ for low-risk CRP in the adult nontransplanted population, but we applied the lower cutoff point $(>0.5 \mathrm{mg} / \mathrm{dL})$ for CRP as we used higher quartile of enrolled populations [26].

Interest in $\mathrm{CV}$ outcomes after kidney transplantation is likely to increase because long-term graft outcomes have improved. The current study revealed that ACS were significantly high in the higher MIA score group 

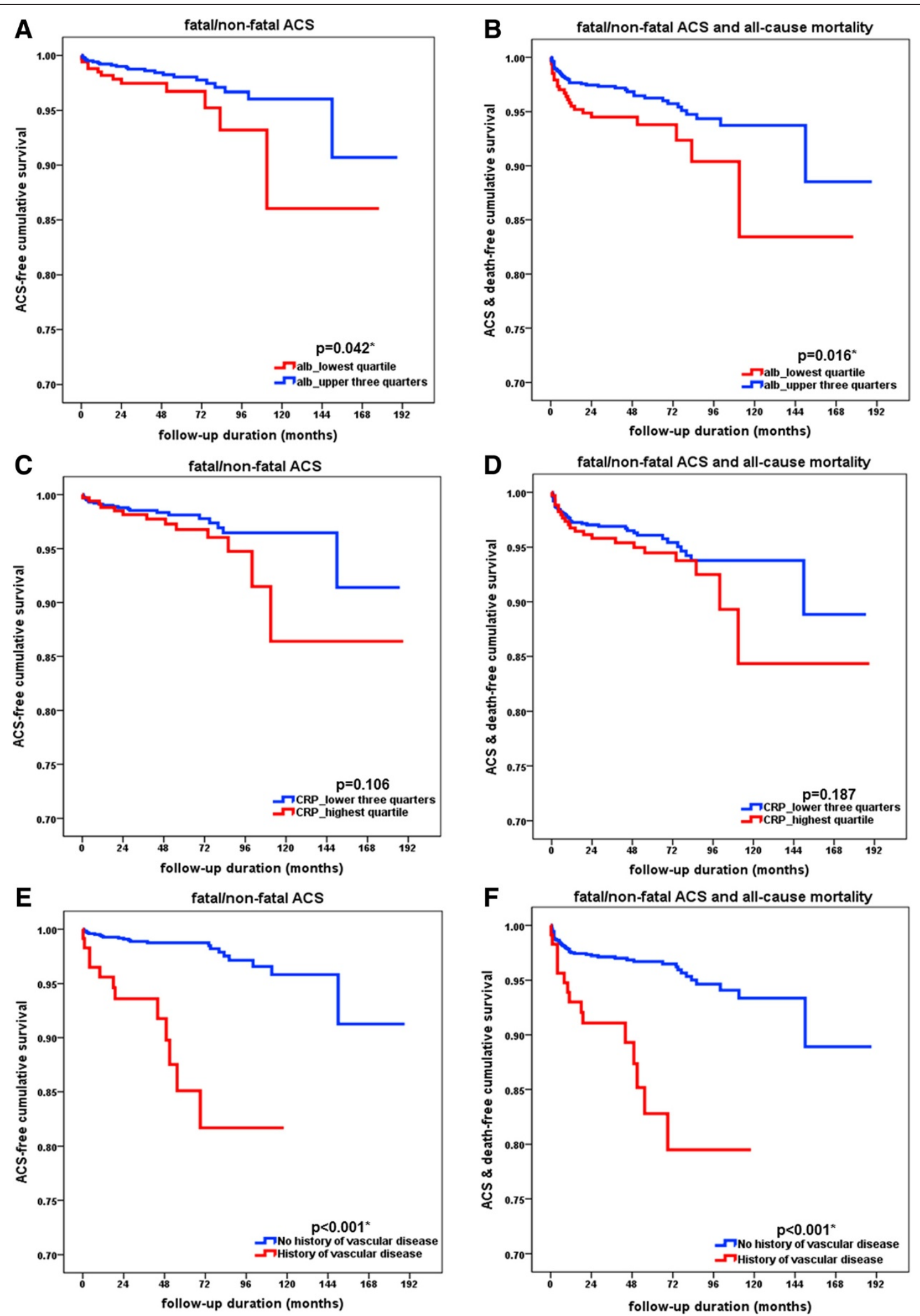

Fig. 2 (See legend on next page.) 
(See figure on previous page.)

Fig. 2 Kaplan-Meier curves of fatal/non-fatal acute coronary syndrome (ACS) and composite outcomes of ACS and death in patients with different MIA factors. $\mathbf{a}-\mathbf{b}$. The patients in the lowest albumin quartile showed worse ACS outcomes $(p=0.042)$ and composite outcomes of ACS and death $(p=0.016)$ than the others. $\mathbf{c}-\mathbf{d}$. The patients in the highest CRP quartile showed tendency of worse ACS outcomes $(p=0.106)$ and composite outcomes of ACS and death $(p=0.187)$ although statistically insignificant. e-f. The patients with previous history of vascular disease showed poor outcome of ACS $(p<0.001)$ and composite outcomes of ACS and death $(p<0.001)$ than the others

compared to the lower MIA score group after multivariate analysis by adjusting possible factors with association. Increasing patient age, DM, male sex, hypertension, dyslipidemia, and cigarette smoking are well known risk factors for CV disease in general [27]. Non-traditional risk factors such as dialysis vintage before transplantation, rejection, anemia, proteinuria, reduced kidney function following transplantation, and elevated inflammatory markers have been associated with increased $\mathrm{CV}$ risk in various studies $[28,29]$. Pretransplant CV disease is the most important predictor of post-transplant CV events [30]. Further, both pretransplant diabetes and NODAT are associated with

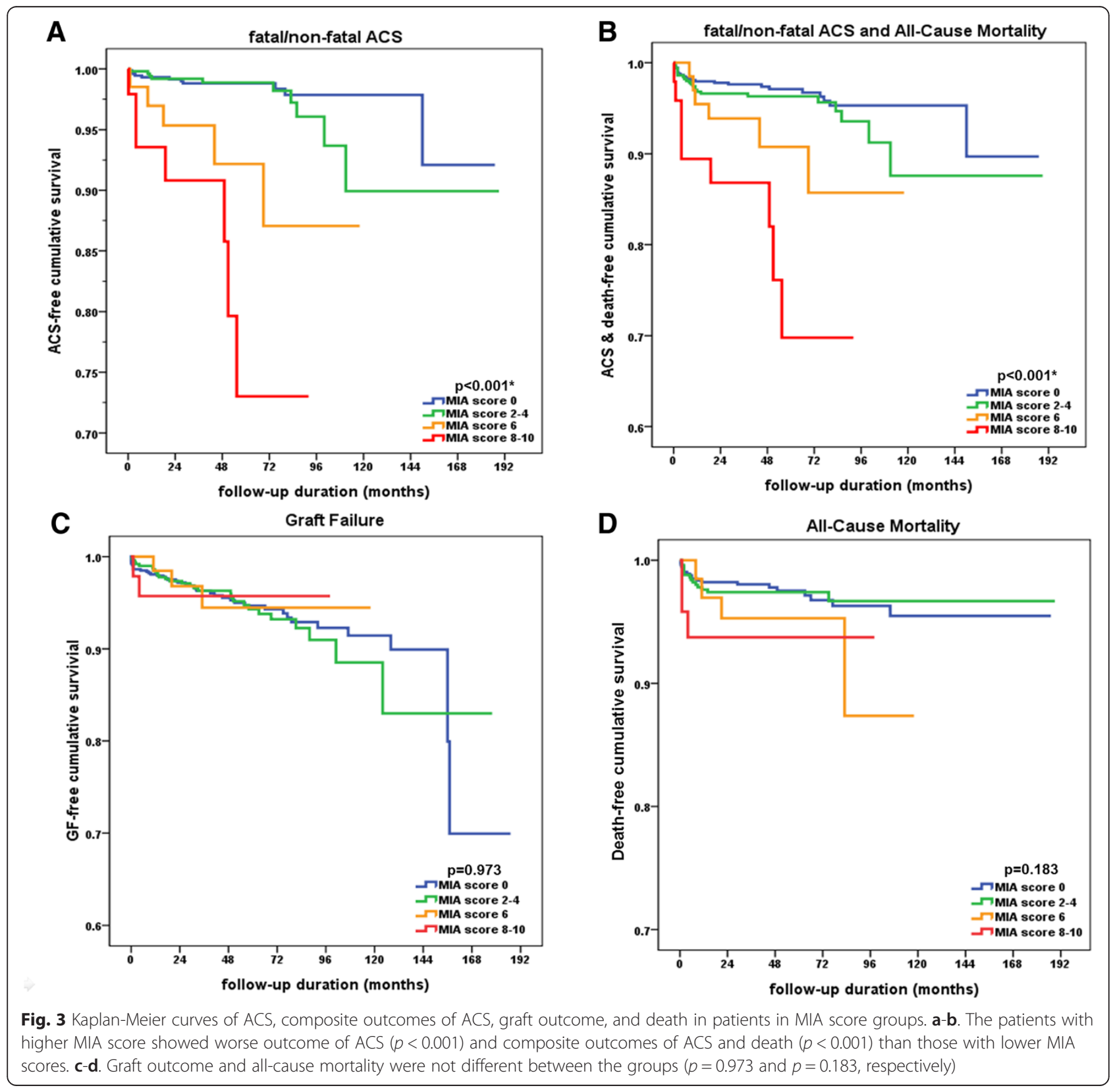




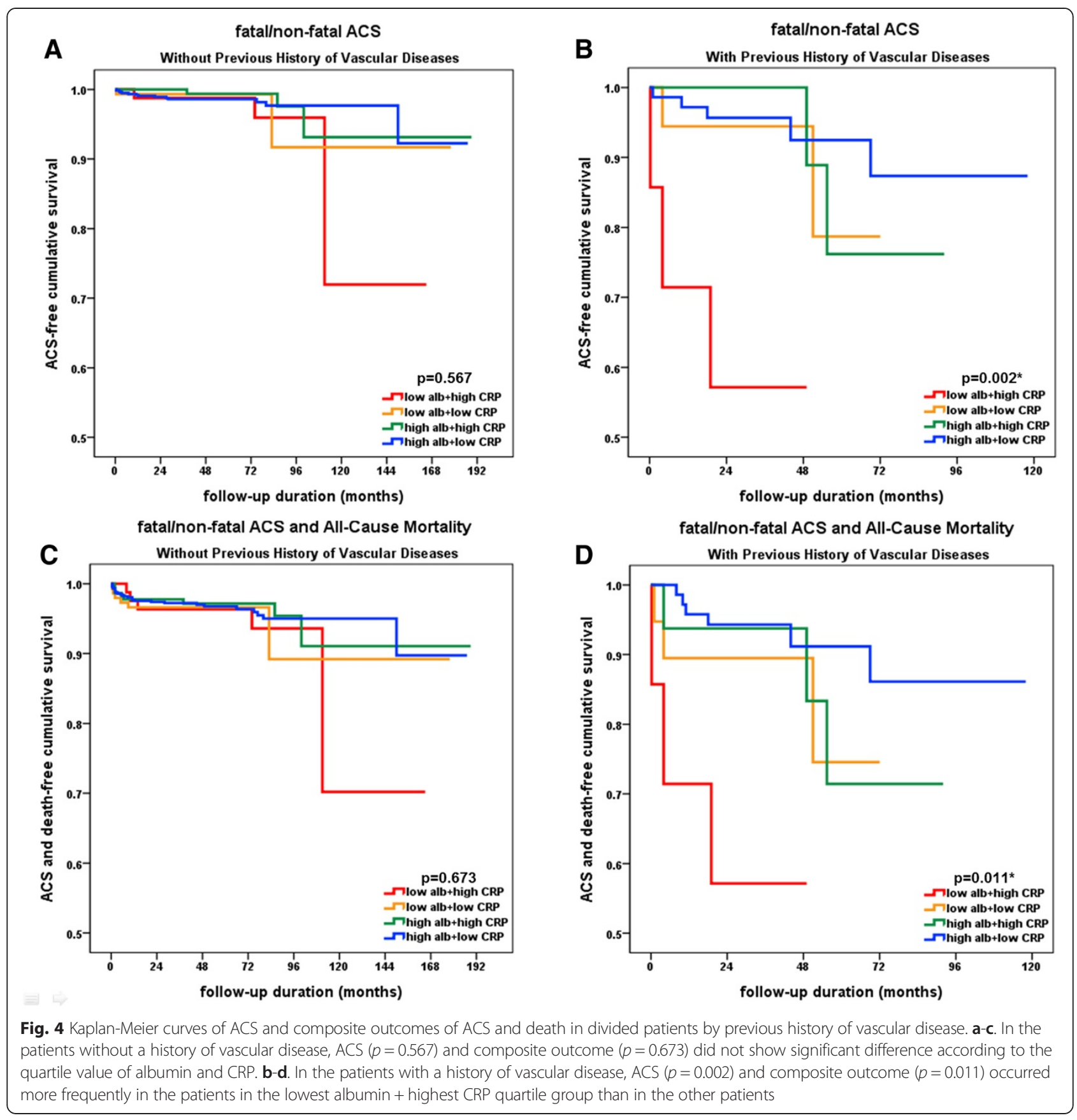

the occurrence of post-transplant CV outcomes [31]. Our results are meaningful because we showed the independent association between the pretransplant MIA score group with ACS after all known risk factors were adjusted.

Our data showed significant result only in ACS and composite outcome. Graft function and other risk factors are known to be associated with cardiovascular outcome [32]. However, in this study, graft failure did not affect the occurrence of fatal/non-fatal ACS.

In this study, pretransplant CRP level did not show independent association with post-transplant ACS, although several former studies reported an association between high CRP levels and CV outcomes [18, 24, 29]. The occurrence of ACS in the patients with sustained high CRP levels after transplantation cannot be verified, because post-transplant one-year CRP value was not measured routinely in all patients.

The overall mortality rate was only $3.0 \%$ in this study. The population was too small to analyze statistical significance and to affect the composite outcome with ACS. For the other aspect, patients with a history of vascular disease are usually selected more carefully 
before entering the waiting list for KT. This could be another reason why the mortality rate was not different with others. Graft failure also failed to show a difference between groups.

The nutritional status of Korean might be different with a Western population and the risk of coronary heart disease is lower in Korean population [33]. The CVD is not the primary cause of death even though the mortality of unknown cause (19.5\%) was assumed as $\mathrm{CV}$ death. To the best of our knowledge, our study is notable for enrolling the largest number of Korean patients in multicenter to provide the analysis for the correlation between the pretransplant MIA factors and ACS. Enrolled clinical institutions are active centers for transplantation in South Korea. We evaluated patients with relatively simple standards that can be easily applied. Also, we used cardiovascular outcome as "ACS", which is specific outcome of ischemic heart disease. The term 'major adverse cardiac event' was commonly used for several studies, but it is considered as inconsistent and of heterogeneous definition [34].

The following limitations should be considered when interpreting our results. Like all other retrospective studies, this study cannot prove a causal relationship. There could be inevitable biases which are patient selection, follow-up, or interpretation of the outcomes. However, we tried to minimize the possible biases by considerate medical record review and by including every factor which can possibly affect the outcome of patients. Also, it was not able to perform the true assessment of nutrition such as subjective global assessment of nutritional status. The method used to calculate the MIA score might need further validation even which was published previously in other fields [19]. The CRP was not a routine laboratory test before and after transplantation in many clinical institutions, and patients who did not have pretransplant serum CRP levels measured were excluded from analysis. Moreover, transferrin is a known marker of malnutrition in patients with ESRD [35], but we could not evaluate transferrin as a malnutrition marker. As the population of this study is all Asian, the results are not directly comparable with US or European cohorts. The heterogeneity of the populations (transplantation types and preemptive KT vs. dialysis before $\mathrm{KT}$ ) can be the other limitation of this study although we verified such clinical findings were not different between the MIA score groups. Lastly, we applied our own standard for classification because there is no standard method to assess malnutrition and inflammation in patients with ESRD.

\section{Conclusions}

We have shown that the presence of higher MIA score before kidney transplantation is an independent predictor of post-transplant ACS. Further investigations are needed to assess whether improving MIA syndrome would impact the occurrence of ACS after kidney transplantation and whether active evaluation and treatment for coronary artery disease would be helpful for improving outcome in patients with high MIA score.

\section{Abbreviations}

CVD: Cardiovascular disease; ESRD: end-stage renal disease;

MIA: Malnutrition, inflammation, and atherosclerosis; GFR: glomerular filtration rate; MICS: malnutrition inflammation complex syndrome; NODAT: new onset diabetes after transplantation; CRP: C-reactive protein; BMI: body mass index; DM: diabetes mellitus; ACS: acute coronary syndrome; Ml: myocardial infarction; HR: hazard ratio; Cl: confidence interval; PTH: parathyroid hormone; CMV: cytomegalovirus.

\section{Competing interests}

The authors declare that they have no competing interests.

\section{Authors' contributions}

All authors presented in this paper contributed extensively to the work at all stages. JHH collected the data, analyzed the results, performed the study, and wrote the manuscript. JR, JNA, CTK, HK performed statistical analyses and interpreted the data analyses. JY, JH, DWC, CA, IMJ, YKO, CSL, DJH, SKP, and YSK collected the data in each centers and analyzed the results. YHK and JPL designed the study, analyzed the results, and wrote and edited the manuscript. All authors read and approved the final manuscript.

\section{Acknowledgements}

This study has no funding source. We thank Nara Shin who helped with data collection.

\section{Author details}

'Department of Internal Medicine, Chung-Ang University Hospital, Seoul, South Korea. ${ }^{2}$ Department of Internal Medicine, Hallym University College of Medicine, Chuncheon, South Korea. ${ }^{3}$ Department of Internal Medicine, Seoul National University Boramae Medical Center, Seoul, South Korea. ${ }^{4}$ Graduate School of Public Health, Seoul National University, Seoul, South Korea. ${ }^{5}$ Department of Internal Medicine, Asan Medical Center and University of Ulsan College of Medicine, Seoul, South Korea. ${ }^{6}$ Transplantation Center, Seoul National University Hospital, Seoul, South Korea. ${ }^{7}$ Department of Internal Medicine, Seoul National University Bundang Hospital, Seongnam, South Korea. ${ }^{8}$ Department of Internal Medicine, Seoul National University College of Medicine, Seoul, South Korea. ${ }^{9}$ Department of Surgery, Seoul National University Boramae Medical Center, Seoul, South Korea. ${ }^{10}$ Department of Surgery, Asan Medical Center and University of Ulsan College of Medicine, Seoul, South Korea.

Received: 4 March 2015 Accepted: 6 July 2015

Published online: 21 July 2015

\section{References}

1. Collins AJ. Cardiovascular mortality in end-stage renal disease. Am J Med Sci. 2003;325(4):163-7.

2. Foley RN, Parfrey PS, Sarnak MJ. Clinical epidemiology of cardiovascular disease in chronic renal disease. Am J Kidney Dis. 1998;32(5 Suppl 3):S112-9.

3. Ishimura E, Shoji T, Emoto M, Motoyama K, Shinohara K, Matsumoto N, et al. Renal insufficiency accelerates atherosclerosis in patients with type 2 diabetes mellitus. Am J Kidney Dis. 2001;38(4 Suppl 1):S186-90.

4. Jungers P, Massy ZA, Nguyen Khoa T, Fumeron C, Labrunie M, Lacour B, et al. Incidence and risk factors of atherosclerotic cardiovascular accidents in predialysis chronic renal failure patients: a prospective study. Nephrol Dial Transplant. 1997;12(12):2597-602.

5. Coresh J, Longenecker JC, Miller ER, Young HJ, Klag MJ. Epidemiology of cardiovascular risk factors in chronic renal disease. J Am Soc Nephrol. 1998;9(12 Suppl):S24-30.

6. Stenvinkel P, Heimburger O, Paultre F, Diczfalusy U, Wang T, Berglund L, et al. Strong association between malnutrition, inflammation, and atherosclerosis in chronic renal failure. Kidney Int. 1999;55(5):1899-911. 
7. Stenvinkel $P$, Heimburger $O$, Lindholm B, Kaysen GA, Bergstrom J. Are there two types of malnutrition in chronic renal failure? Evidence for relationships between malnutrition, inflammation and atherosclerosis (MIA syndrome). Nephrol Dial Transplant. 2000;15(7):953-60.

8. Lowrie EG, Lew NL. Death risk in hemodialysis patients: the predictive value of commonly measured variables and an evaluation of death rate differences between facilities. Am J Kidney Dis. 1990;15(5):458-82.

9. Pecoits-Filho $R$, Lindholm B, Stenvinkel P. The malnutrition, inflammation, and atherosclerosis (MIA) syndrome - the heart of the matter. Nephrol Dial Transplant. 2002;17 Suppl 11:28-31.

10. Caglar K, Peng Y, Pupim LB, Flakoll PJ, Levenhagen D, Hakim RM, et al. Inflammatory signals associated with hemodialysis. Kidney Int. 2002;62(4):1408-16.

11. Raj DS, Dominic EA, Pai A, Osman F, Morgan M, Pickett G, et al. Skeletal muscle, cytokines, and oxidative stress in end-stage renal disease. Kidney Int. 2005;68(5):2338-44.

12. Stenvinkel $P$. Inflammatory and atherosclerotic interactions in the depleted uremic patient. Blood Purif. 2001;19(1):53-61.

13. Fishbane S. Cardiovascular risk evaluation before kidney transplantation. J Am Soc Nephrol. 2005;16(4):843-5.

14. Patel RK, Mark PB, Johnston N, McGeoch R, Lindsay M, Kingsmore DB, et al Prognostic value of cardiovascular screening in potential renal transplant recipients: a single-center prospective observational study. Am J Transplant. 2008;8(8):1673-83.

15. Kalantar-Zadeh K, Kopple JD, Block G, Humphreys MH. A malnutritioninflammation score is correlated with morbidity and mortality in maintenance hemodialysis patients. Am J Kidney Dis. 2001;38(6):1251-63.

16. Molnar MZ, Czira ME, Rudas A, Ujszaszi A, Lindner A, Fornadi K, et al. Association of the malnutrition-inflammation score with clinical outcomes in kidney transplant recipients. Am J Kidney Dis. 2011;58(1):101-8.

17. Molnar MZ, Kovesdy CP, Bunnapradist S, Streja E, Mehrotra R, Krishnan M, et al. Associations of pretransplant serum albumin with post-transplant outcomes in kidney transplant recipients. Am J Transplant. 201 1;11(5):1006-15.

18. Varagunam M, Finney $H$, Trevitt $R$, Sharples E, McCloskey DJ, Sinnott PJ, et al. Pretransplantation levels of $\mathrm{C}$-reactive protein predict all-cause and cardiovascular mortality, but not graft outcome, in kidney transplant recipients. Am J Kidney Dis. 2004;43(3):502-7.

19. Ay H, Gungor L, Arsava EM, Rosand J, Vangel M, Benner T, et al. A score to predict early risk of recurrence after ischemic stroke. Neurology. 2010;74(2):128-35.

20. Fink JC, Onuigbo MA, Blahut SA, Christenson RH, Mann D, Bartlett ST, et al. Pretransplant serum C-reactive protein and the risk of chronic allograft nephropathy in renal transplant recipients: a pilot case-control study. Am J Kidney Dis. 2002;39(5):1096-101.

21. Perez RV, Brown DJ, Katznelson SA, Dubin JA, Muller HG, Chang T, et al. Pretransplant systemic inflammation and acute rejection after renal transplantation. Transplantation. 2000;69(5):869-74.

22. van Ree RM, Oterdoom LH, de Vries AP, Gansevoort RT, van der Heide JJ, van Son WJ, et al. Elevated levels of C-reactive protein independently predict accelerated deterioration of graft function in renal transplant recipients. Nephrol Dial Transplant. 2007;22(1):246-53.

23. Winkelmayer WC, Schaeffner ES, Chandraker A, Kramar R, Rumpold H, Sunder-Plassmann G, et al. A J-shaped association between high-sensitivity C-reactive protein and mortality in kidney transplant recipients. Transpl Int. 2007;20(6):505-11

24. Kruger B, Walberer A, Debler J, Boger CA, Farkas S, Reinhold SW, et al. Is inflammation prior to renal transplantation predictive for cardiovascular and renal outcomes? Atherosclerosis. 2010;210(2):637-42.

25. Kuhlmann MK, Kribben A, Wittwer M, Hörl WH. OPTA—malnutrition in chronic renal failure. Nephrol Dial Transplant. 2007;22 suppl 3:iii13-iii9.

26. Pearson TA, Mensah GA, Alexander RW, Anderson JL, Cannon 3rd RO, Criqui M et al. Markers of inflammation and cardiovascular disease: application to clinical and public health practice: A statement for healthcare professionals from the Centers for Disease Control and Prevention and the American Heart Association. Circulation. 2003;107(3):499-511.

27. Farquhar JW, Fortmann SP, Flora JA, Taylor CB, Haskell WL, Williams PT, et al. Effects of communitywide education on cardiovascular disease risk factors. The Stanford Five-City Project. JAMA. 1990;264(3):359-65.

28. Ducloux D, Kazory A, Chalopin JM. Predicting coronary heart disease in renal transplant recipients: a prospective study. Kidney Int. 2004;66(1):441-7.

29. Abedini S, Holme I, Marz W, Weihrauch G, Fellstrom B, Jardine A, et al. Inflammation in renal transplantation. Clin J Am Soc Nephrol. 2009;4(7):1246-54.
30. Gill JS, Ma I, Landsberg D, Johnson N, Levin A. Cardiovascular events and investigation in patients who are awaiting cadaveric kidney transplantation. J Am Soc Nephrol. 2005;16(3):808-16.

31. Lentine KL, Brennan DC, Schnitzler MA. Incidence and predictors of myocardial infarction after kidney transplantation. J Am Soc Nephrol. 2005;16(2):496-506.

32. Forsythe JL. Graft function and other risk factors as predictors of cardiovascular disease outcome. Transplantation. 2001;72(6 Suppl):S16-9.

33. Jee $\mathrm{SH}$, Jang $\mathrm{Y}, \mathrm{O}$ Oh DJ, Oh BH, Lee SH, Park SW, et al. A coronary heart disease prediction model: the Korean Heart Study. BMJ Open. 2014;4(5), e005025.

34. Kip KE, Hollabaugh K, Marroquin OC, Williams DO. The problem with composite end points in cardiovascular studies: the story of major adverse cardiac events and percutaneous coronary intervention. J Am Coll Cardiol. 2008;51(7):701-7.

35. Molnar MZ, Keszei A, Czira ME, Rudas A, Ujszaszi A, Haromszeki B, et al. Evaluation of the malnutrition-inflammation score in kidney transplant recipients. Am J Kidney Dis. 2010;56(1):102-11.

\section{Submit your next manuscript to BioMed Central and take full advantage of:}

- Convenient online submission

- Thorough peer review

- No space constraints or color figure charges

- Immediate publication on acceptance

- Inclusion in PubMed, CAS, Scopus and Google Scholar

- Research which is freely available for redistribution 